Copyright (C) 2002 IFAC

15th Triennial World Congress, Barcelona, Spain

\title{
THE GUARANTEED COST CONTROL FOR UNCERTAIN LARGE-SCALE INTERCONNECTED SYSTEMS
}

\author{
Hiroaki Mukaidani* Yasuyuki Takato* \\ Yoshiyuki Tanaka* Koich Mizukami ${ }^{* *}$ \\ * Faculty of Information Sciences, Hiroshima City University, \\ 3-4-1, Ozukahigashi Asaminami-ku Hiroshima, 731-3194 Japan. \\ e-mail:mukaida@im.hiroshima-cu.ac.jp \\ ** Faculty of Engineering, Hiroshima Kokusai Gakuin University, \\ 6-20-1, Nakano Aki-ku Hiroshima, 739-0321 Japan.
}

\begin{abstract}
The guaranteed cost control problem of the decentralized robust control for a class of large-scale interconnected systems with norm-bounded time-varying parameter uncertainties is considered. Based on the LMI design approach, a class of decentralized local state feedback controllers is proposed, and some sufficient conditions for the existence of guaranteed cost controllers are derived by making use of the LMI.
\end{abstract}

Keywords: Large-scale interconnected systems, Decentralized control, Guaranteed cost control, LMI, Parameter uncertainties

\section{INTRODUCTION}

The study of large-scale interconnected systems has received ever greater attention in the past few decades (see, for example, Siljak, 1978 and the references therein). In recent years, the problem of the decentralized robust control of large-scale systems with parameter uncertainties has been widely studied, and some solution approaches have been developed (Wang et al., 1997; Guo et al., 2000; Yan et al., 1998; Wang et al., 1998; Gong et al., 1996; Xie and Xie, 2000; Zhang et al., 1996). In Guo et al. (2000) the decentralized control problem for the uncertain interconnected timevarying systems which do not satisfy the so-called matching conditions has been studied. In Wang et al. (1997) and Guo et al. (2000), a decentralized stabilizing nonlinear state feedback controller has been proposed for the nonlinear multimachine power systems via the algebraic Riccati equation (ARE) approach. Furthermore, in Wang et al. (1998), the results developed in Wang et al. (1997) have been extended to the class of large-scale interconnected nonlinear systems via the robust decentralized linear control. Compared with the nonlinear controllers, there exists an important feature that the linear controllers are of simpler structure and easier to be implemented. However, in case where we apply the ARE approach, if some additional requirements such as the minimization of the upper bound on the value of the cost function are added, there exists the drawback for solving such specific problems. Furthermore, how to select the optimal multiparameters which are included in the AREs has never been studied.

Although there have been numerous results on decentralized robust control of large-scale uncertain systems, much effort has been made towards finding a controller which guarantees robust stability. However, when controlling such systems, it is also desirable to design the control systems which guarantee not only the robust stability, but also an adequate level of performance. One approach to this problem is the so-called guaranteed cost control approach (Petersen and MacFarlane 
1994). This approach has the advantage of providing an upper bound on a given performance index. Recent advance in theory of linear matrix inequality (LMI) has allowed a revisiting of the guaranteed cost control approach. In particular, the guaranteed cost control problem for a class of nonlinear large-scale interconnected systems which is based on the LMI design method was solved (Xie et al., 2000). The LMI design method is a very well-known and powerful tool, it can not only efficiently find feasible and global solutions, but also easily handle various kinds of additional linear constraints. However, so far the problem of guaranteed cost stabilization for the large-scale uncertain linear systems with respect to uncertainties in the controllers themselves has not been discussed.

In this paper, the guaranteed cost control problem (Petersen and MacFarlane 1994) which has received much attention recently of the decentralized robust control for a class of large-scale systems with the norm-bounded parameter uncertainties is considered. After defining the guaranteed cost control problem for the large-scale interconnected uncertain systems, a sufficient condition for the existence of the decentralized robust feedback controllers is derived by making use of the Lyapunov stability criterion such that uncertain large-scale interconnected systems can be asymptotically stabilized. The LMI design approach plays an important role to derive such sufficient conditions. The main contributions of this paper show that the guaranteed cost controllers can be constructed by solving the LMI. It is worth pointing out that the positive scaling parameters which are included in the LMI are chosen such that an upper bound on the quadratic cost performance is optimized. Consequently, the resulting controllers guarantee the adequate upper bound on a given performance. Finally, the problem of guaranteed cost control for large-scale uncertain systems under gain perturbations is considered.

\section{PROBLEM FORMULATION}

Consider a class of large-scale interconnected systems composed of $N$ interconnected subsystems described by the following state equations:

$$
\begin{aligned}
\dot{x}_{i}(t)= & {\left[A_{i}+\Delta A_{i}(t)\right] x_{i}(t)+\left[B_{i}+\Delta B_{i}(t)\right] u_{i}(t) } \\
& +\sum_{j=1, j \neq i}^{N} G_{i j} g_{i j}\left(t, x_{j}\right), \\
x_{i}(0)= & x_{i 0}, \quad i=1,2, \cdots, N,
\end{aligned}
$$

where $x_{i} \in \mathbf{R}^{n_{i}}$ and $u_{i} \in \mathbf{R}^{m_{i}}$ are the state and control of the $i$ th subsystems, respectively. $A_{i}$, $B_{i}$ and $G_{i j}$ are constant matrices of appropriate dimensions and $G_{i j}$ are interconnection matrices between the $i$ th subsystems and other subsystems. The unknown vector functions $g_{i j}\left(t, x_{j}\right) \in \mathbf{R}^{l_{i}}$ represent interconnections among the subsystems. It is assumed that the unknown vector functions $g_{i j}\left(t, x_{j}\right)$ are continuous and sufficiently smooth in $x_{j}$ and piecewise continuous in $t$ (Gong et al., 1996). The parameter uncertainties considered here are assumed to be of the following form:

$$
\left[\Delta A_{i}(t) \Delta B_{i}(t)\right]=D_{i} F_{i}(t)\left[\begin{array}{ll}
E_{1 i} & E_{2 i}
\end{array}\right],
$$

where $D_{i}, E_{1 i}$ and $E_{2 i}$ are known constant real matrices of appropriate dimensions.

$F_{i}(t) \in \mathbf{R}^{p_{i} \times q_{i}}$ are unknown matrix functions with Lebesgue measurable elements and satisfying $F_{i}^{T}(t) F_{i}(t) \leq I_{q_{i}}$, where $I_{l} \in \mathbf{R}^{l \times l}$ denote the identity matrices.

We make the following assumptions concerning the unknown vector functions.

Assumption 1: There exists known constant matrix $W_{i j}$ such that for all $x_{j} \in \mathbf{R}^{n_{j}}$

$$
\left\|g_{i j}\left(t, x_{j}\right)\right\| \leq\left\|W_{i j} x_{j}\right\|,
$$

for all $i, j$ and for all $t \geq 0$, where $\|\cdot\|$ denotes the Euclidean norm.

Assumption 2: For all $i, \sum_{j=1}^{N} W_{j i}^{T} W_{j i}>0$.

Remark 1: The assumption 2 is made only for simplification of presentation.

Associated with system (1) is the cost function

$$
J=\sum_{i=1}^{N} \int_{0}^{\infty}\left[x_{i}^{T}(t) Q_{i} x_{i}(t)+u_{i}^{T}(t) R_{i} u_{i}(t)\right] d t
$$

where $Q_{i}$ and $R_{i}$ are given positive definite symmetric matrices.

Definition: A control law $u_{i}(t)=K_{i} x_{i}(t)$ is said to be a quadratic guaranteed cost control with cost matrix $P_{i}>0$ for uncertain large-scale interconnected systems (1) and cost function (4) if the closed-loop systems are quadratically stable and the closed-loop value of the cost function (4) satisfies the bound $J \leq \bar{J}$ for all admissible uncertainties, that is,

$$
\begin{aligned}
\sum_{i=1}^{N}( & \frac{d}{d t} x_{i}^{T}(t) P_{i} x_{i}(t) \\
& \left.+x_{i}^{T}(t)\left[Q_{i}+K_{i}^{T} R_{i} K_{i}\right] x_{i}(t)\right)<0
\end{aligned}
$$

for all nonzero $x_{i} \in \mathbf{R}^{n_{i}}$ and all matrices $F_{i}(t)$ : $F_{i}^{T}(t) F_{i}(t) \leq I_{q_{i}}$. 
The objective of this paper is to design a decentralized linear time-invariant guaranteed cost control law $u_{i}(t)=K_{i} x_{i}(t), i=1,2, \cdots, N$ for the large-scale interconnected systems (1) with uncertainties (2).

\section{MAIN RESULTS}

Now, we present a sufficient condition for existence of the state feedback guaranteed cost control laws for the uncertain system (1).

Theorem 1: Under the assumptions 1 and 2, let us consider the large-scale interconnected systems (1) with the uncertainties (2). If there exist symmetric positive definite matrices $P_{i} \in \mathbf{R}^{n_{i} \times n_{i}}$ such that for all uncertain matrices $F_{i}(t)$ the LMI (6) is satisfied, the control laws $u_{i}(t)=K_{i} x_{i}(t), i=$ $1,2, \cdots, N$ are the guaranteed cost controller,

$$
\Lambda_{i}=\left[\begin{array}{cccc}
\Xi_{i} & P_{i} G_{i 1} & \cdots & P_{i} G_{i N} \\
G_{i 1}^{T} P_{i} & -I_{l_{i}} & \cdots & 0 \\
\vdots & \vdots & \ddots & \vdots \\
G_{i N}^{T} P_{i} & 0 & \cdots & -I_{l_{i}}
\end{array}\right]<0
$$

where $\Lambda_{i} \in \mathbf{R}^{\bar{N} \times \bar{N}}, \bar{N}=n_{i}+(N-1) l_{i}$ and

$$
\begin{aligned}
& \Xi_{i}:=\tilde{A}_{i}^{T} P_{i}+P_{i} \tilde{A}_{i}+\sum_{j=1, j \neq i}^{N} W_{j i}^{T} W_{j i}+\bar{R}_{i}, \\
& \tilde{A}_{i}:=\bar{A}+D_{i} F_{i}(t) \bar{E}_{i}, \bar{A}_{i}:=A_{i}+B_{i} K_{i}, \\
& \bar{E}_{i}:=E_{1 i}+E_{2 i} K_{i}, \bar{R}_{i}:=Q_{i}+K_{i}^{T} R_{i} K_{i} .
\end{aligned}
$$

Furthermore, the corresponding value of the cost function (4) satisfies the following inequality (7) for all admissible uncertainties $F_{i}(t)$.

$$
J<\bar{J}=\sum_{i=1}^{N} x_{i}^{T}(0) P_{i} x_{i}(0) .
$$

Remark 1. Note that there exists no matrix $P_{i} G_{i i}$, $i=1, \cdots, N$ in the matrix $\Lambda_{i}$.

Proof: Combining the guaranteed cost controller $u_{i}(t)=K_{i} x_{i}(t)$ with (1) gives a closed-loop system of the form

$$
\dot{x}_{i}=\tilde{A} x_{i}+\sum_{j=1, j \neq i}^{N} G_{i j} g_{i j}\left(t, x_{j}\right) .
$$

Suppose now there exist symmetric positive definite matrices $P_{i}>0$ such that the LMI (6) holds for all admissible uncertainties. In order to prove the asymptotic stability of the closed-loop system (8), let the Lyapunov function candidate

$$
V(x(t))=\sum_{i=1}^{N} x_{i}^{T}(t) P_{i} x_{i}(t)
$$

where $x=\left[\begin{array}{llll}x_{1}^{T} & x_{2}^{T} & \cdots & x_{N}^{T}\end{array}\right]^{T}$. Note that $V(x)>0$ whenever $x \neq 0$. Then the time derivative of $V(x)$ along any trajectory of the closed-loop system (8) is given by

$$
\begin{aligned}
\frac{d}{d t} V(x)= & \sum_{i=1}^{N} z_{i}^{T}\left[\begin{array}{cccc}
\Xi_{i}-\bar{R}_{i} & P_{i} G_{i 1} & \cdots & P_{i} G_{i N} \\
G_{i 1}^{T} P_{i} & -I_{l_{i}} & \cdots & 0 \\
\vdots & \vdots & \ddots & \vdots \\
G_{i N}^{T} P_{i} & 0 & \cdots & -I_{l_{i}}
\end{array}\right] z_{i} \\
& -\sum_{i=1}^{N} \sum_{j=1, j \neq i}^{N}\left(x_{j}^{T} W_{i j}^{T} W_{i j} x_{j}-g_{i j}^{T} g_{i j}\right) \\
= & \sum_{i=1}^{N} z_{i}^{T} \Lambda_{i} z_{i}-\sum_{i=1}^{N} x_{i}^{T} \bar{R}_{i} x_{i} \\
& -\sum_{i=1}^{N} \sum_{j=1, j \neq i}^{N}\left(x_{j}^{T} W_{i j}^{T} W_{i j} x_{j}-g_{i j}^{T} g_{i j}\right),
\end{aligned}
$$

where $z_{i}=\left[\begin{array}{lllll}x_{i}^{T} & g_{i 1}^{T} & g_{i 2}^{T} & \cdots & g_{i N}^{T}\end{array}\right]^{T} \in \mathbf{R}^{\bar{N}}$ and $\Xi_{i}$ and $\Lambda_{i}$ are given in (6). Taking into account the fact that the inequalities (6) hold, and using the Schur complement (Zhou, 1998), it follows immediately that

$$
\frac{d}{d t} V(x)<-\sum_{i=1}^{N} x_{i}^{T} \bar{R}_{i} x_{i}<0 .
$$

Hence, $V(x)$ is a Lyapunov function for the closed-loop system (8). Therefore, the closed-loop system (8) is asymptotically stable and $u_{i}(t)=$ $K_{i} x_{i}(t)$ is the guaranteed cost controller because the inequality (5) is satisfied. Furthermore, by integrating both sides of the inequality (10) from 0 to $T$ and using the initial conditions, we have

$$
V(x(T))-V(x(0))<-\sum_{i=1}^{N} \int_{0}^{T} x_{i}^{T} \bar{R}_{i} x_{i} d t .
$$

Since the closed-loop system (8) is asymptotically stable, that is, $x_{i}(T) \rightarrow 0$, when $T \rightarrow \infty$, we obtain $V(x(T)) \rightarrow 0$. Thus we get

$$
\begin{aligned}
J & =\sum_{i=1}^{N} \int_{0}^{T} x_{i}^{T} \bar{R}_{i} x_{i} d t<V(x(0)) \\
& =\sum_{i=1}^{N} x_{i}^{T}(0) P_{i} x_{i}(0)=\bar{J}
\end{aligned}
$$

It follows from the definition that the result of the theorem is true. The proof of Theorem 1 is completed.

We now give the LMI design approach to the construction of a guaranteed cost controller.

Theorem 2: Under the assumptions 1 and 2, suppose there exist the constant parameters $\mu_{i}>0$ 
such that for all $i=1,2, \cdots, N$ the following LMI (11) have a symmetric positive definite matrix $X_{i}>0 \in \mathbf{R}^{n_{i} \times n_{i}}$ and a matrix $Y_{i} \in \mathbf{R}^{m_{i} \times n_{i}}$

$$
\left[\begin{array}{ccccc}
\Phi_{i} & \tilde{E}_{i}^{T} & X_{i} & Y_{i}^{T} & X_{i} \\
\tilde{E}_{i} & -\mu_{i} I_{n_{i}} & 0 & 0 & 0 \\
X_{i} & 0 & -Q_{i}^{-1} & 0 & 0 \\
Y_{i} & 0 & 0 & -R_{i}^{-1} & 0 \\
X_{i} & 0 & 0 & 0 & -U_{i}^{-1}
\end{array}\right]<0,(11)
$$

where $\tilde{E}_{i}:=E_{1 i} X_{i}+E_{2 i} Y_{i}, U_{i}:=\sum_{j=1, j \neq i}^{N} W_{j i}^{T} W_{j i}$,

$$
\begin{aligned}
\Phi_{i}:= & A_{i} X_{i}+B_{i} Y_{i}+\left(A_{i} X_{i}+B_{i} Y_{i}\right)^{T}+\mu_{i} D_{i} D_{i}^{T} \\
& +\sum_{j=1, j \neq i}^{N} G_{i j} G_{i j}^{T} .
\end{aligned}
$$

Then, the decentralized linear state feedback control laws

$u_{i}(t)=K_{i} x_{i}(t)=Y_{i} X_{i}^{-1} x_{i}(t), i=1, \cdots, N,(12)$

are the guaranteed cost control laws and

$$
J<\sum_{i=1}^{N} x_{i}^{T}(0) X_{i}^{-1} x_{i}(0)=\bar{J}
$$

is the guaranteed cost for the closed-loop uncertain large-scale interconnected systems.

Proof: Applying the Schur complement to the LMI (11) gives

$$
\begin{aligned}
& \Phi_{i}+\left[\left(E_{1 i} X_{i}+E_{2 i} Y_{i}\right)^{T} X_{i} Y_{i}^{T} X_{i}\right] \\
& +\left[\begin{array}{cccc}
\mu_{i}^{-1} I_{n_{i}} & 0 & 0 & 0 \\
0 & Q_{i} & 0 & 0 \\
0 & 0 & R_{i} & 0 \\
0 & 0 & 0 & U_{i}
\end{array}\right]\left[\begin{array}{c}
E_{1 i} X_{i}+E_{2 i} Y_{i} \\
X_{i} \\
Y_{i} \\
X_{i}
\end{array}\right] \\
= & A_{i} X_{i}+B_{i} Y_{i}+\left(A_{i} X_{i}+B_{i} Y_{i}\right)^{T}+\mu_{i} D_{i} D_{i}^{T} \\
& +\sum_{j=1, j \neq i}^{N} G_{i j} G_{i j}^{T} \\
& +\mu_{i}^{-1}\left(E_{1 i} X_{i}+E_{2 i} Y_{i}\right)^{T}\left(E_{1 i} X_{i}+E_{2 i} Y_{i}\right) \\
& +X_{i} Q_{i} X_{i}+Y_{i}^{T} R_{i} Y_{i}+X_{i} U_{i} X_{i}<0 .
\end{aligned}
$$

Let us introduce the matrices $X_{i}=P_{i}^{-1}$ and $Y_{i}=K_{i} P_{i}^{-1}$. Substituting these matrices into the above inequality (14) and pre- and postmultiplying both sides of the inequality (14) by $P_{i}$ yield

$$
\begin{aligned}
\Gamma_{i} & :=\bar{A}_{i}^{T} P_{i}+P_{i} \bar{A}_{i}+\mu_{i} P_{i} D_{i} D_{i}^{T} P_{i} \\
& +\mu_{i}^{-1} \bar{E}_{i}^{T} \bar{E}_{i}+P_{i}\left(\sum_{j=1, j \neq i}^{N} G_{i j} G_{i j}^{T}\right) P_{i} \\
& +\sum_{j=1, j \neq i}^{N} W_{j i}^{T} W_{j i}+\bar{R}_{i}<0 .
\end{aligned}
$$

We will use these inequalities in order to establish (6). For any admissible uncertainties (2), it follows from (15) and a standard matrix inequality (Petersen and MacFarlane 1994) that

$$
\begin{aligned}
& \tilde{A}_{i}^{T} P_{i}+P_{i} \tilde{A}_{i}+P_{i}\left(\sum_{j=1, j \neq i}^{N} G_{i j} G_{i j}^{T}\right) P_{i} \\
& +\sum_{j=1, j \neq i}^{N} W_{j i}^{T} W_{j i}+\bar{R}_{i} \leq \Gamma_{i} .
\end{aligned}
$$

On the other hand, by applying the Schur complement, it is easy to verify that the above inequality is equivalent to $(6)$.

Since the LMI (11) consists of a convex solution set of $\left(\mu_{i}, X_{i}, Y_{i}\right)$, various efficient convex optimization algorithm can be applied. Moreover, its solutions parameterize the set of the guaranteed cost controllers. This parameterized representation can be exploited to design the guaranteed cost controllers which minimizes the value of the guaranteed cost for the closed-loop uncertain large-scale systems. Consequently, solving the following optimization problem allows us to determine the optimal bound.

$$
\Sigma_{0}: \min _{\mathcal{X}_{i}} \sum_{i=1}^{N} \alpha_{i}=J^{*}, \mathcal{X}_{i} \in\left(\mu_{i}, X_{i}, Y_{i}, \alpha_{i}\right)(16)
$$

such that (11) and

$$
\left[\begin{array}{cc}
-\alpha_{i} & x_{i}^{T}(0) \\
x_{i}(0) & -X_{i}
\end{array}\right]<0
$$

That is, the problem addressed in this paper is as follows: "Find $K_{i}=Y_{i} X_{i}^{-1}, i=1,2, \cdots, N$ such that LMI (11) and (17) are satisfied and the cost $\bar{J}<\sum_{i=1}^{N} \alpha_{i}$ becomes as small as possible."

Thus, the minimization of $\bar{J}$ implies the minimization of the guaranteed cost for the uncertain large-scale systems (1). Finally, we are in a position to establish the main result of this section.

Theorem 3: If the above optimization problem has the solution $\mu_{i}, X_{i}, Y_{i}$ and $\alpha_{i}$, then the control laws of the form (12) are the decentralized linear optimal state feedback control laws which ensure the minimization of the guaranteed cost $\bar{J}$ for the uncertain large-scale interconnected systems.

Proof: By Theorem 2, the control laws (12) constructed from the feasible solutions $\mu_{i}, X_{i}, Y_{i}$ and $\alpha_{i}$ are the guaranteed cost controllers of the uncertain large-scale interconnected systems (1). Using the Schur complement to the LMI (17), we have 


$$
\left[\begin{array}{cc}
-\alpha_{i} & x_{i}^{T}(0) \\
x_{i}(0) & -X_{i}
\end{array}\right]<0 \Leftrightarrow x_{i}^{T}(0) X_{i}^{-1} x_{i}(0)<\alpha_{i} .
$$

It follows that

$$
J<\sum_{i=1}^{N} x_{i}^{T}(0) X_{i}^{-1} x_{i}(0)<\min _{\mathcal{X}_{i}} \sum_{i=1}^{N} \alpha_{i}=J^{*} .
$$

Thus, the minimization of $\sum_{i=1}^{N} \alpha_{i}$ implies the minimization of the guaranteed cost $\bar{J}$ for the interconnected uncertain systems (1). The optimality of the solution of the optimization problem follows from the convexity of the objective function under the LMI constraints. This is the required result.

Remark 2: It can be noted that the bound obtained in Theorem 3 depends on the initial condition $x_{i}(0)$. To remove this dependence on $x_{i}(0)$, we assume that $x_{i}(0)$ is a zero mean random variable satisfying $E\left[x_{i}(0) x_{i}(0)^{T}\right]=I_{n_{i}}$, where $E[\cdot]$ denotes the expectation. In this case, it should be pointed out that the guaranteed cost becomes $E[J]=\sum_{i=1}^{N} E\left[x_{i}^{T}(0) X_{i}^{-1} x_{i}(0)\right]=\sum_{i=1}^{N} \operatorname{Trace} X_{i}^{-1}$ $<\sum_{i=1}^{N}$ Trace $\mathcal{A}_{i}$ such that (11) and

$$
\left[\begin{array}{cc}
-\mathcal{A}_{i} & I_{n_{i}} \\
I_{n_{i}} & -X_{i}
\end{array}\right]<0
$$

Furthermore, it is worth pointing out that the original optimization problem for the guaranteed cost (16) can be decomposed to the reduced optimization problems (19) because each optimization problem (19) is independent of other LMI. Hence, we have only to solve the optimization problems (19) for each independent subsystem.

$$
\Sigma_{i}: \min _{\mathcal{Y}_{i}} \text { Trace } \mathcal{A}_{i}, \mathcal{Y}_{i} \in\left(\mu_{i}, X_{i}, Y_{i}, \mathcal{A}_{i}\right) .(19)
$$

\section{GUARANTEED COST CONTROL UNDER ADDITIVE GAIN PERTURBATIONS}

Consider the class of large-scale interconnected systems (1), where $E_{2 i} \equiv 0$. For a given controllers $u_{i}(t)=K_{i} x_{i}(t)$, the actual controller implemented is assumed to be $u_{i}(t)=\left[K_{i}+\right.$ $\left.H_{i} F_{i}(t) E_{i}^{k}\right] x_{i}(t)$, where $K_{i}$ is the nominal controller gain, and $H_{i} F_{i}(t) E_{i}^{k}$ represents the gain perturbations. In fact, the controller gain perturbations can result from the actuator degradations, as well as from the requirement for re-adjustment of controller gains during the controller implementation stage (see e.g. Yang et al., 2000).

By using the similar algebraic technique used in Theorem 2, we give the following theorem.
Theorem 4: Under the assumptions 1 and 2, suppose there exist the constant parameters $\mu_{i}>0$ such that for all $i=1,2, \cdots, N$ the following LMI (20) have a symmetric positive definite matrix $X_{i}>0 \in \mathbf{R}^{n_{i} \times n_{i}}$ and a matrix $Y_{i} \in \mathbf{R}^{m_{i} \times n_{i}}$

$\left[\begin{array}{ccccc}\Psi_{i} & X_{i} & X_{i} & Y_{i}^{T}+B_{i} \tilde{H}_{i}^{\mu} & X_{i} \hat{E}_{i}^{T} \\ X_{i} & -Q_{i}^{-1} & 0 & 0 & 0 \\ X_{i} & 0 & -U_{i}^{-1} & 0 & 0 \\ Y_{i}+\tilde{H}_{i}^{\mu} B_{i}^{T} & 0 & 0 & -R_{i}^{-1}+\tilde{H}_{i}^{\mu} & 0 \\ \hat{E}_{i} X_{i} & 0 & 0 & 0 & -\mu_{i} I_{n_{i}}\end{array}\right]$

$<0$,

where $\hat{E}_{i}:=\left[\begin{array}{ll}E_{1 i}^{T} & E_{i}^{k T}\end{array}\right]^{T}, \tilde{H}_{i}^{\mu}:=\mu_{i} H_{i} H_{i}^{T}$

$\Psi_{i}:=A_{i} X_{i}+B_{i} Y_{i}+\left(A_{i} X_{i}+B_{i} Y_{i}\right)^{T}+\mu_{i}\left(D_{i} D_{i}^{T}\right.$

$\left.+B_{i} H_{i} H_{i}^{T} B_{i}^{T}\right)+\sum_{j=1, j \neq i}^{N} G_{i j} G_{i j}^{T}$

$U_{i}:=\sum_{j=1, j \neq i}^{N} W_{j i}^{T} W_{j i}>0$.

Then, the decentralized linear state feedback control laws (12) are the guaranteed cost control laws and the cost bounds (13) is the guaranteed cost for the closed-loop uncertain large-scale interconnected systems.

Proof: Since it is easy to prove this theorem by applying the Schur complement and the standard matrix inequality (Petersen and MacFarlane 1994), the proof is omitted.

\section{NUMERICAL EXAMPLE}

In order to demonstrate the efficiency of our proposed control, we have run a simple numerical examples. Consider the interconnected uncertain systems (1) composed of three two-dimensional subsystems. The system matrices and the unknown functions with the uncertainties are given as follows.

$$
\begin{aligned}
& A_{1}=\left[\begin{array}{cc}
0 & 1 \\
-1 & 0.01
\end{array}\right], B_{1}=\left[\begin{array}{l}
0 \\
1
\end{array}\right], D_{1}=\left[\begin{array}{l}
0 \\
1
\end{array}\right], \\
& G_{12}=\left[\begin{array}{c}
0 \\
0.2
\end{array}\right], G_{13}=\left[\begin{array}{c}
0 \\
0.1
\end{array}\right], x_{1}=\left[\begin{array}{l}
x_{11} \\
x_{12}
\end{array}\right], \\
& A_{2}=\left[\begin{array}{cc}
0 & 1 \\
-2 & -3
\end{array}\right], B_{2}=\left[\begin{array}{l}
0 \\
2
\end{array}\right], D_{2}=\left[\begin{array}{c}
0 \\
1.5
\end{array}\right], \\
& G_{23}=\left[\begin{array}{c}
0 \\
0.4
\end{array}\right], G_{21}=\left[\begin{array}{c}
0 \\
0.1
\end{array}\right], x_{2}=\left[\begin{array}{l}
x_{21} \\
x_{22}
\end{array}\right], \\
& A_{3}=\left[\begin{array}{cc}
0 & 1 \\
1 & 0
\end{array}\right], B_{3}=\left[\begin{array}{c}
0 \\
0.5
\end{array}\right], D_{3}=\left[\begin{array}{c}
0 \\
0.5
\end{array}\right], \\
& G_{31}=\left[\begin{array}{c}
0 \\
0.3
\end{array}\right], G_{32}=\left[\begin{array}{c}
0 \\
0.2
\end{array}\right], x_{3}=\left[\begin{array}{l}
x_{31} \\
x_{32}
\end{array}\right], \\
& E_{11}=E_{12}=E_{13}=\left[\begin{array}{ll}
0 & 0.1
\end{array}\right], \\
& E_{21}=E_{22}=E_{23}=\left[\begin{array}{ll}
0.01
\end{array}\right], \\
& g_{1 j}=\left[0.1+\delta_{1}(t)\right] \sin \left[\begin{array}{ll}
1 & 0
\end{array}\right] x_{j}, j=2,3, \\
& g_{2 j}=\left[0.1+\delta_{2}(t)\right] \sin \left[\begin{array}{ll}
1 & 0
\end{array}\right] x_{j}, j=3,1, \\
& g_{3 j}=\left[0.1+\delta_{3}(t)\right] \sin \left[\begin{array}{ll}
1 & 0
\end{array}\right] x_{j}, j=1,2,
\end{aligned}
$$




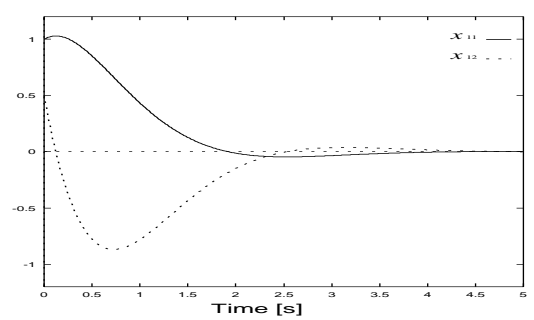

Fig. 1. Response of the closed-loop system 1.

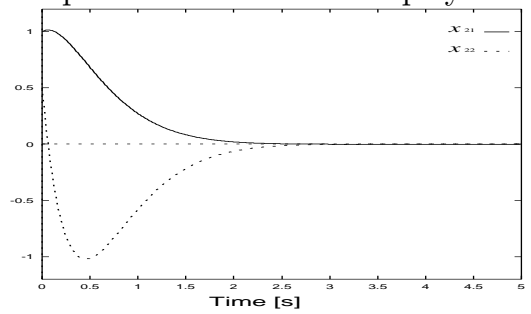

Fig. 2. Response of the closed-loop system 2.

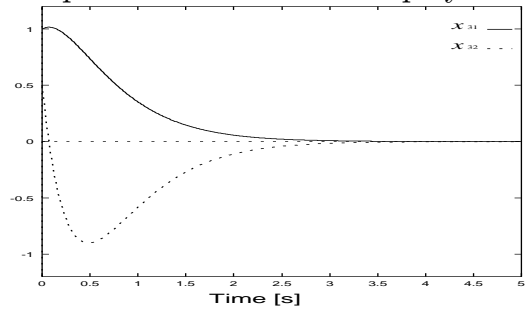

Fig. 3. Response of the closed-loop system 3.

$$
\left|\delta_{i}(t)\right| \leq 0.1, i=1,2,3,
$$

In that case the unknown functions $g_{i j}\left(t, x_{j}\right)$ satisfy $\left\|g_{i j}\left(t, x_{j}\right)\right\| \leq 0.2\left\|\sin \left[\begin{array}{ll}1 & 0\end{array}\right] x_{j}\right\| \leq 0.2\left\|x_{j}\right\|$. Therefore, we choose as $W_{12}=W_{13}=W_{23}=$ $W_{21}=W_{31}=W_{32}=0.2 I_{2}$. The uncertain parameters $F_{i}(t), i=1,2,3$ are time-variant and satisfy $\left|F_{i}(t)\right| \leq 1$. Now, we choose as $R_{i}=1$ and $Q_{i}=\operatorname{diag}[10.01 .0], i=1,2$, 3. By applying Theorem 3 and solving the corresponding optimization problem (19), we obtain the decentralized linear optimal state feedback control laws

$$
\begin{aligned}
& K_{1}=[-2.4071-2.6079], \\
& K_{2}=\left[-2.4177-9.4040 \times 10^{-1}\right], \\
& K_{3}=[-1.0667 \times 10-9.7170] .
\end{aligned}
$$

Consequently, the guaranteed cost of the uncertain closed-loop system is $J^{*}=66.71748$, where minTrace $\mathcal{A}_{1}=10.857576$, minTrace $\mathcal{A}_{2}=$ 7.068615 and $\operatorname{\mathcal {Y}}_{\mathcal{Y}_{3}}$ Trace $\mathcal{A}_{3}=48.791289$. Finally, the results of the simulation of this example are depicted in Fig. 1-3. The initial state is set as $x_{i}(0)=\left[\begin{array}{ll}1 & 0.5\end{array}\right]^{T}, i=1,2,3$. It is shown from Fig. 1-3 that the closed-loop system is asymptotically stable.

\section{CONCLUSIONS}

In this paper, a solution of the guaranteed cost control problem for large-scale interconnected systems with the norm-bounded parameter uncertainties has been presented. The decentralized robust optimal guaranteed cost controller which minimizes the value of the guaranteed cost for the closed-loop uncertain large-scale systems can be solved by using software such as MATLAB's LMI control Toolbox. Thus, the resulting decentralized linear feedback controller can guarantee the quadratic stability and the optimal cost bound for the uncertain large-scale systems.

\section{References}

Gong Z., C. Wen and D. P. Mital (1996). Decentralized robust controller design for a class of interconnected uncertain systems: with unknown bound of uncertainty, IEEE Trans. Automat. Contr., 41, 850-854.

Guo Y., D. J. Hill and Y. Wang (2000). Nonlinear decentralized control of large-scale power systems, Automatica, 36, 1275-1289.

Petersen I. R. and D. C. McFarlane (1994) Optimal guaranteed cost control and filtering for uncertain linear systems, IEEE Trans. Automat. Contr., 39, 1971-1977.

Siljak, D. D., (1978). Large-Scale Dynamic Systems: Stability and Structure, Amsterdam: North Holland.

Wang, Y., G. Guo and D. J. Hill (1997). Robust decentralized non-linear controller design for multimachine power systems, Automatica, 33, 1725-1733.

Wang Y., D. J. Hill and G. Guo (1998). Robust decentralized control for multimachine power systems, IEEE Trans. Circuits and Systems, 45, 271-279.

Xie S. and L. Xie (2000). Stabilization of a class of uncertain large-scale stochastic systems with time delays, Automatica, 36, 161-167.

Xie S., L. Xie, T. Wang and G. Guo (2000). Decentralized control of multimachine power systems with guaranteed performance, IEE Proc. Control Theory Appl., 147, 355-365.

Yan X.-G. and G.-Z. Dai (1998). Decentralized output feedback robust control for nonlinear large-scale systems, Automatica, 34, 14691472.

Yang G.-H. J. L. Wang and Y. C. Soh (2000). Guaranteed cost control for discrete-time linear systems under controller gain perturbations, Linear Algebra and its Applications, 312, 161180.

Zhang S. Y., K. Mizukami and H. S. Wu (1996). Decentralized robust control for a class of uncertain large-scale interconnected nonlinear dynamical systems, J. Opt. Theory and Applications, 91, 235-256.

Zhou K. (1998). Essentials of Robust Control, New Jersey: Prentice-Hall. 